\title{
Phase diagram and polarization of stable phases of $\left(\mathrm{Ga}_{1-x} \operatorname{In}_{x}\right)_{2} \mathrm{O}_{3}$
}

\author{
Maria Barbara Maccioni and Vincenzo Fiorentini \\ Department of Physics, University of Cagliari and CNR-IOM, \\ UOS Cagliari, Cittadella Universitaria, 09042 Monserrato (CA), Italy
}

\begin{abstract}
Using density-functional ab initio calculations, we provide a revised phase diagram of $\left(\mathrm{Ga}_{1-x} \operatorname{In}_{x}\right)_{2} \mathrm{O}_{3}$. Three phases - monoclinic, hexagonal, cubic bixbyite- compete for the ground state. In particular, in the $x \sim 0.5$ region we expect coexistence of hexagonal, $\beta$, and bixbyite (the latter separating into binary components). Over the whole $x$ range, mixing occurs in three disconnected regions, and non-mixing in two additional distinct regions. We then explore the permanent polarization of the various phases, finding that none of them is polar at any concentration, despite the possible symmetry reductions induced by alloying. On the other hand, we find that the $\varepsilon$ phase of $\mathrm{Ga}_{2} \mathrm{O}_{3}$ stabilized in recent growth experiments is pyroelectric -i.e. locked in a non-switchable polarized structure- with ferroelectric-grade polarization and respectable piezoelectric coupling. We suggest that this phase could be used profitably to produce high-density electron gases in transistor structures.

PACS numbers: 61.66.Dk,77.22.Ej,81.05.Zx
\end{abstract}

\section{INTRODUCTION}

The Ga and In sesquioxides have recently been under intense scrutiny as, among others, UV absorbers and trasparent conductors. With a view at exploiting materials engineering concepts from other semiconductor systems, a growing body of work is being devoted to the $\left(\mathrm{Ga}_{1-x} \mathrm{In}_{x}\right)_{2} \mathrm{O}_{3}$ alloy. Theoretical studies on its phase stability and optical properties have been published recently by at least two groups 15 , but the picture is apparently still far from complete. Recent growth experiments $\sqrt{617}$ on the $\left(\mathrm{Ga}_{1-x} \mathrm{In}_{x}\right)_{2} \mathrm{O}_{3}$ alloy in the vicinity of $x=0$ and $x=0.5$ have suggested that phases other than those so far assumed as ground state may in fact be stable or stabilized by constrained (e.g. epitaxial) growth. In one paper ${ }^{6}$, three competing phases are reported to appear near $x \sim 0.5$ : a hexagonal phase previously observed at exactly $x=0.5$; a monoclinic close relative of the $\beta-\mathrm{Ga}_{2} \mathrm{O}_{3}$ structure; and that derived from the bixbyite structure of $\operatorname{In}_{2} \mathrm{O}_{3}$, mostly in the form of phase-separated In and $\mathrm{Ga}$ oxides. Another paper ${ }^{7}$ reported that the $\varepsilon$ phase of $\mathrm{Ga}_{2} \mathrm{O}_{3}$ can be obtained at $820 \mathrm{~K}$ via epitaxial growth on $\mathrm{GaN}$, although a bulk phase transition to that phase from the ground state $\beta$ phase is not expected below $1500 \mathrm{~K}^{8}$.

In this work, we report $i$ ) a phase diagram of $\left(\mathrm{Ga}_{1-x} \mathrm{In}_{x}\right)_{2} \mathrm{O}_{3}$ accounting for new findings around $x \simeq 0.5$, and $i i$ ) the polarization of the competing phases, plus the $\varepsilon-\mathrm{Ga}_{2} \mathrm{O}_{3}$ phase. The results are in a nutshell that a) the hex and $\beta$ structures do indeed compete energetically with the bixbyite phase expected based on previous results, and this competition occurs predominantly in the vicinity of $x \sim 0.5$, giving rise to a fairly complex phase diagram with interlacing mixing and non-mixing concentration regions; $b$ ) none of the alloy phases is polar, but the $\varepsilon$ phase of $\mathrm{Ga}_{2} \mathrm{O}_{3}$ is. As dictated by its symmetry, this phase (only slightly energy-disfavored over the stable $\beta$ phase) has a large spontaneous polarization and sizable piezoelectric coupling. Importantly, it cannot be transformed into (is not symmetry-related to) the stable $\beta$ phase. These results open up some interesting perspectives, such as growing the hex phase epitaxially, or exploiting the polarization properties of $\varepsilon-\mathrm{Ga}_{2} \mathrm{O}_{3}$.

\section{METHODS}

Geometry and volume optimizations as well as electronic structure calculations are performed using densityfunctional theory (DFT) in the generalized gradient approximation (GGA), and the Projector Augmented-Wave (PAW) method as implemented in the VASP code ${ }^{9}$. In all calculations the cut-off is $471 \mathrm{eV}$ and the force threshold is $0.01 \mathrm{eV} / \AA$. For all phases, 80 -atom cells are used. The k-point summation grids are a $\Gamma$-centered $2 \times 2 \times 2$ for the hexagonal phase and $4 \times 4 \times 2$ for the $\varepsilon$ phase $(4 \times 4 \times 2$ for the $\beta$ phase and $4 \times 4 \times 4$ for the bixbyite structure as in Ref 3). The polarization is calculated via the Berryphase approach, $\frac{\sqrt{10}}{1}$ the $\mathrm{k}$-grid is a $4 \times 4$ set of 16 -point strings.

Phase coexistence is determined by the Helmholtz mixing free energy per cation (the enthalpy vanishes because the pressure is kept to zero to numerical accuracy in all calculations) of the mixture as a function of $x$,

$$
F_{\text {mix }}=F_{\text {alloy }}-F_{\text {bulk }}=\left[E_{\text {alloy }}-T S_{\text {alloy }}\right]-F_{\text {bulk }},
$$

where $E_{\text {alloy }}$ is the internal energy calculated from first principles as just described, and

$$
\begin{aligned}
S_{\text {alloy }}= & -x \log x-(1-x) \log (1-x)+ \\
& +3[(1+n) \log (1+n)-n \log n]=S_{\text {mix }}+S_{\text {vib }}
\end{aligned}
$$

with

$$
\begin{aligned}
n(T, x) & =1 /\left(e^{\Theta_{m}(x) / T}-1\right), \\
\Theta_{m}(x) & =(1-x) \Theta_{\mathrm{Ga}_{2} \mathrm{O}_{3}}+x \Theta_{\mathrm{In}_{2} \mathrm{O}_{3}}
\end{aligned}
$$

the Planck distribution and the mixture's Debye temperature $\Theta_{m}(x)$ interpolated between the parent compounds. (The approximation of the vibrational entropy 
with that of a single-Debye-frequency oscillator is admissible, as the growth temperatures are comparable to or higher than the Debye temperatures $\Theta_{\mathrm{In}_{2} \mathrm{O}_{3}}=420 \mathrm{~K}$ and $\Theta_{\mathrm{Ga}_{2} \mathrm{O}_{3}}=870 \mathrm{~K}$.) The bulk free energy

$$
F_{\text {bulk }}(x)=x F_{\mathrm{In}_{2} \mathrm{O}_{3}}+(1-x) F_{\mathrm{Ga}_{2} \mathrm{O}_{3}}
$$

interpolates between the binary-compound values, calculated as for the alloy. We finally recall that a mixture separates into phases if the specific free energy is a negative-curvature function of $x$. The $x$ values where the curvature becomes negative and, respectively, goes back to positive (i.e. the inflection points of the mixing free energy) delimit the phase separation region; these bounds, which may depend on temperature $\mathrm{T}$, define a range known as miscibility gap.

\section{RESULTS}

\section{A. Hexagonal phase near $x \simeq 0.5$}

We first consider a hexagonal phase of $\left(\mathrm{Ga}_{1-x} \mathrm{In}_{x}\right)_{2} \mathrm{O}_{3}$. The motivation comes from recent growth experiments 6 of $\left(\mathrm{Ga}_{1-x} \mathrm{In}_{x}\right)_{2} \mathrm{O}_{3}$ near $x \sim 0.5$, which revealed the appearance, besides $\beta$ and bixbyite crystal portions, of significant hexagonal microcrystallites. A candidate phase had been identified earlier on ${ }^{11}$ for $\mathrm{InGaO}_{3}$, and classified in the non-polar space group $P 6_{3} / m m c$. This phase is depicted at $x=0.5$ in Fig.1.
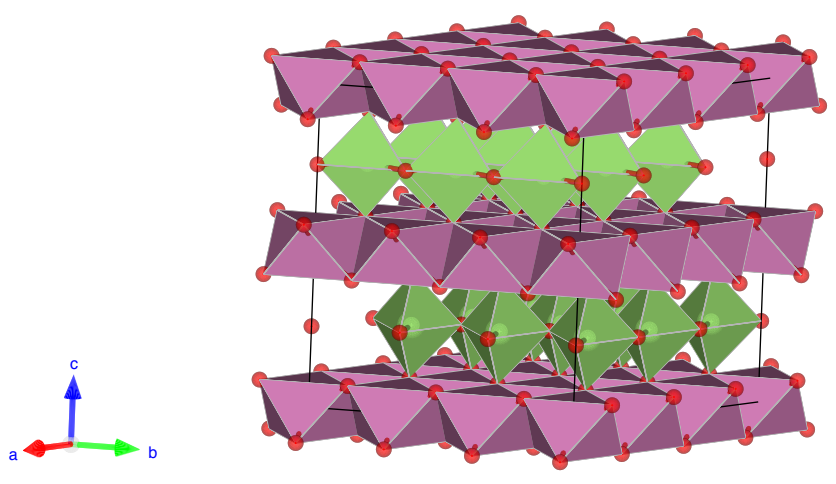

FIG. 1. The hex structure at 50-50 concentration.

Both out of interest for its possible energetic stability, and for the possibility that the structure might become polar, we investigated this phase in the range $x \in[0.45,0.55]$. In this region the hex phase is lower in energy than, and therefore favored over, the bixbyite and virtually degenerate at $x=0.5$ with the $\beta$ phase (discussed below). We quantify this calculating the Helmholtz mixing free energy by the model described above and in Ref.3. (As in previous work, $\frac{1}{3}$ large error bars are expected from the limited configurational sampling, but as errors should largely cancel out when comparing the various phases, we deem the relative energetics to be rather reliable.) As shown in Fig 2 bottom panel, we find that the lowest energy structures of the sample of configurations for the hex symmetry in the vicinity of $x=0.5$ are lower in mixing free energy by about $0.1 \mathrm{eV}$ than the freestanding bixbyite configurational sample, and therefore more stable than the bixbyite alloy.

\section{B. $\beta$ phase near $x \sim 0.5$}

In previous work ${ }^{1 / 3}$ we found that the alloy adopting the $\beta$ structure of $\mathrm{Ga}_{2} \mathrm{O}_{3}$ is disfavored over bixbyite for $x$ above 0.1 or so. The internal energy of that phase increases drastically and monotonically in that region of $x$, so we refrained from pursuing it further. However, the same paper reporting the occurrence of hex phase crystallites also signaled $\beta$-phase inclusions near $x=0.5$, so we revisited our previous assessment and studied the $\beta$ phase in that region of concentration. It turns out that at exactly $x=0.5$ the $\beta$ phase is more stable than bixbyite and as stable as the hex phase discussed above (see Fig, 2 , bottom; a similar occurrence was reported in Ref (5). At this concentration, In atoms occupy all the octahedral sites, and Ga atoms occupy all the tetrahedral sites. However, consistently with our previous conclusions, as soon as we move away from exact 50-50 concentration the energy shoots up immediately on both sides of the $x=0.5$ minimum, accompanied by a volume collapse (mainly of the tetrahedra) by over $10 \%$ at $x=0.47$ and $x=0.53$. Therefore, the $\beta$ phase itself should only occur at the "magic" 50-50 concentration, or in the vicinity of that concentration if one assumes that some other phase will take up the local cation excess.

\section{Revised phase diagram}

Based on our calculations discussed above we provide an improved phase diagram accounting for the new phases. The diagram is reported in Fig 2 , bottom panel, as mixing free energy vs $x$. The temperature is $800 \mathrm{~K}$, a typical growth temperature. As shown previously, $\frac{3}{3}$ the phase boundaries are insensitive to temperature within our model, and hence apply to all practical growth temperatures. Put differently, the miscibility gaps and miscibility regions are persistent with temperature.

The stability of the $\beta$ phase only at low $x$ is confirmed, and so is the phase separation into components of the bixbyite phase in most of its own range (signaled by the free energy being everywhere upward-convex except for $x \geq 0.9$ ). What is new is that the hex phase is now the stable one in a range that, conservatively, extends from $x \sim 0.4$ to $x \sim 0.6$. Given its upward-concave free energy, the hex phase does not phase-separate into binary components in this range. In addition, as mentioned, the $\beta$ phase has a very narrow stability slot at $x=0.5$.

As dictated by the curvature of the mixing free energy, there is full miscibility of the two binary oxides at all 


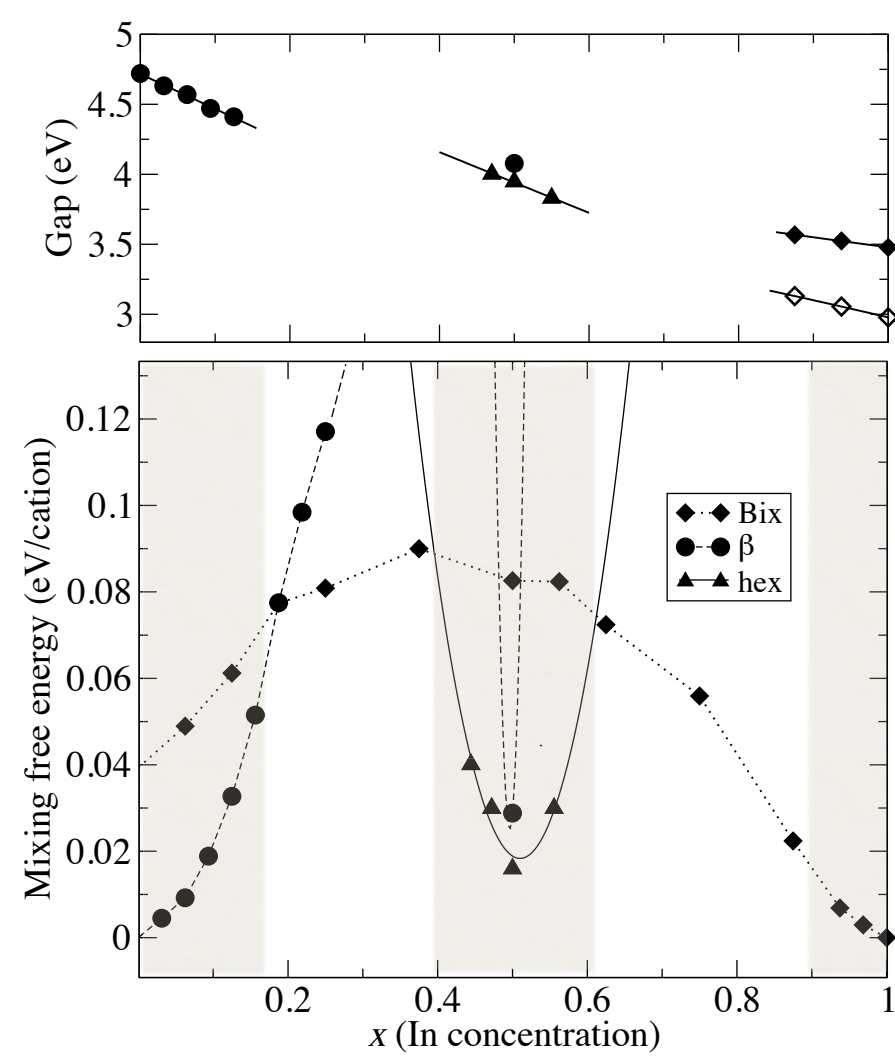

FIG. 2. Revised phase diagram at $\mathrm{T}=800 \mathrm{~K}$ (bottom) and energy gap (top) at $\mathrm{T}=0$ for $\left(\mathrm{Ga}_{1-x} \mathrm{In}_{x}\right)_{2} \mathrm{O}_{3}$. Mixing regions are shaded, and the gap is only drawn for those regions. Legend applies to both panels; empty diamonds: dipole-forbidden transition in bixbyite. The lines are quadratic fits for hex and $x \sim 0.5$, guides to the eye for bixbyite and low $-x \beta$.

temperatures in the ranges $x \in(0,0.18), x \in(0.4,0.6)$, and $x \in(0.9,1)$, where, respectively, the $\beta$, the hex, and the bixbyite structures are adopted. (These $x$ values correspond to the inflection points of the mixing free energy.) In the rest of the $x$ range, separation into binaries is expected from the convex mixing free energy.

In the central region of the $x$ range, there are several competing possibilities. The hex and $\beta$ mixed phases are obviously favored over the bixbyite alloy. But the latter should phase-separate into binary components, with $\mathrm{In}_{2} \mathrm{O}_{3}$ certainly adopting the bixbyite structure. $\mathrm{Ga}_{2} \mathrm{O}_{3}$ may go either bixbyite or $\beta$ : in the first case, the energy (from an interpolation between the end values) is about $0.02 \mathrm{eV}$, i.e. falls between the $\beta$ alloy and the hex; in the second case, the free energy is zero by construction, making phase separation at $50 \%$ slightly favored. These considerations, however, neglect internal interfaces, grain boundaries, strain effects in the binaries, and growth kinetics (all of which are exceedingly complicated and well beyond our present scope), which will tend to disfavor phase separation. Thus, at this level of accuracy, it seems very plausible that -as experiments suggest- the hex, $\beta$, and phase-separated binaries will coexist in this region, depending on the growth conditions.

In Fig 2, top panel, we also report the calculated fundamental gap in the stable phases in the regions where mixing occurs. The gap is calculated as difference of Kohn-Sham GGA eigenvalues plus the empirical correction used in Ref 3 to adjust the gap of the binaries to the experimental values. At low $x$ and at high $x$ one expects optical absorption typical of $\mathrm{Ga}_{2} \mathrm{O}_{3}$ and $\mathrm{In}_{2} \mathrm{O}_{3}$, respectively. Around $x \sim 0.5$, the hex and $\beta$ alloy absorptions should be present; since the bixbyite alloy phase separates, absorption may also be observed at energies typical of $\mathrm{Ga}_{2} \mathrm{O}_{3}(4.5-4.7 \mathrm{eV})$ and $\mathrm{In}_{2} \mathrm{O}_{3}(2.9 \mathrm{eV}$ forbidden, $3.5 \mathrm{eV}$ allowed)); so in the central $x$ region, distinct transitions may be expected at roughly $3.5 \mathrm{eV}, 4 \mathrm{eV}$ and $4.5 \mathrm{eV}$.

\section{Polarization: $\varepsilon-\mathrm{Ga}_{2} \mathrm{O}_{3}$}

One reason of interest in the hex phase is checking whether it distorts into a non-centrosymmetric symmetry group as a consequence of alloying. We investigate a range of alloying of between $43 \%$ and $57 \%$, enabling all symmetry lowerings starting from $P 6_{3} / m m c$. We find that the polarization is always numerically zero referred to the non-polar high-symmetry phase, and are thus forced to conclude that this structure, somewhat anticlimatically, is robustly non polar. In fact, our conclusion agrees with the symmetry determination of Ref.12 at $x=0.5$, and shows that this applies at generic concentrations in that vicinity. We also sampled the polarization in a few bixbyite and $\beta$ alloy samples, unsurprisingly finding them to be always zero (referred to the cubic and monoclinic- $\beta$ phases).

We therefore turned to another potentially polar phase of this system, $\varepsilon-\mathrm{Ga}_{2} \mathrm{O}_{3}$, recently grown epitaxially on GaN by Oshima et al ${ }^{\frac{1}{7}}$. The $\varepsilon$ phase is structurally akin to the same phase of $\mathrm{Fe}_{2} \mathrm{O}_{3}$ and its space group is $P n a 2_{1}$, which does not contain inversion. We calculate its structural parameters, finding them in essential agreement with a previous study,$[8$ and the energy difference with the $\beta$ phase at zero temperature, which is just $90 \mathrm{meV}$ per formula unit.

At low enough temperature, the epitaxial stabilization ${ }^{7}$ of the $\varepsilon$ phase is not endangered by a possible decay in the $\beta$ ground state, for the simple reason that there is no possible $\varepsilon$-to- $\beta$ symmetry path, since the two space groups are $P n a 2_{1}$ and $C 2 / m$, respectively. This is quite analogous to the situation of wurtzite III-V nitrides (group $P 6_{3} m c$ ) which cannot transform, again for symmetry reasons, into the close relative structure of zincblende (group $F \overline{4} 3 m$ ), despite the volume being almost the same and the energy difference being about only $10 \mathrm{meV} /$ atom (the two $\varepsilon$ and $\beta$ phases also have the same volume and an energy difference of about $15 \mathrm{meV} /$ atom). Just as $\varepsilon-\mathrm{Ga}_{2} \mathrm{O}_{3}$, zincblende nitrides can be grown under appropriate epitaxial constraints.

Since the group $P n a 2_{1}$ does not contain inversion, ob- 


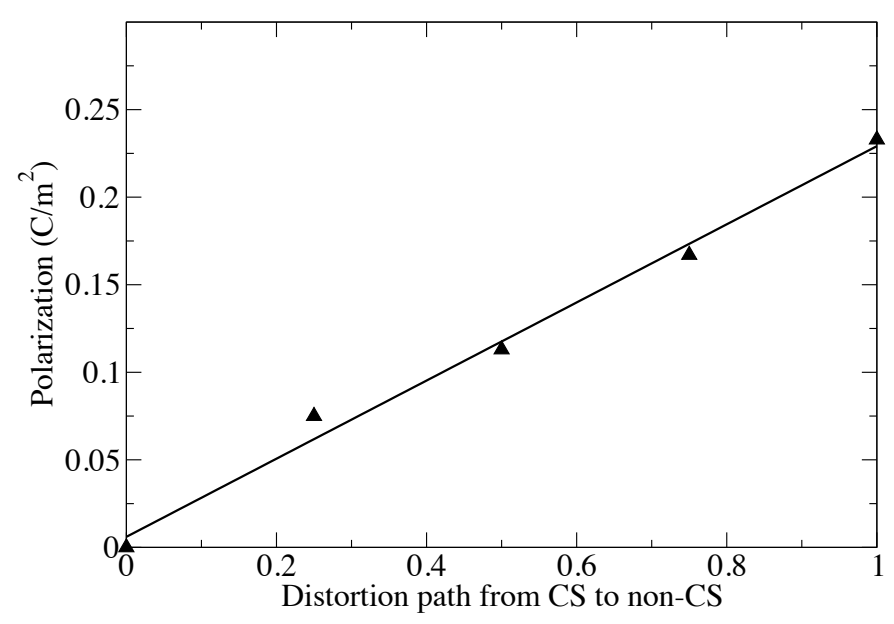

FIG. 3. Polarization calculated along a path connecting the centrosymmetric parent phase to non-centrosymmetric $\varepsilon$ $\mathrm{Ga}_{2} \mathrm{O}$.

servable polar vector quantities are allowed in $\varepsilon-\mathrm{Ga}_{2} \mathrm{O}_{3}$. The polar axis is the $c$ axis, so we calculate the spontaneous polarization $\mathbf{P}=(0,0, \mathrm{P})$ as difference of the polarizations calculated 10 in this phase and in a symmetryconnected centrosymmetric parent phase (we verified that the other components are indeed zero). The latter is chosen to have symmetry group Pnma (a supergroup of $\left.P n a 2_{1}\right)$. The evolution of $\mathrm{P}$ with a path connecting the two structures is in Fig 3 .

The final result is that $\varepsilon-\mathrm{Ga}_{2} \mathrm{O}_{3}$ has a remarkable $\mathrm{P}=0.23 \mathrm{C} / \mathrm{m}^{2}$, a value similar to that of $\mathrm{BaTiO}_{3}$, a factor of 3 larger than of AlN, and nearly a factor 10 larger than of GaN. The structure of the $\varepsilon$ phase is not structurally switchable (in the same sense that wurtzite is not, though of course the polar axis can be inverted, again as in nitrides, by inverting the growth direction using e.g. a buffer layer); therefore $\mathrm{P}$ is expected to mantain its orientation along the polar axis within any given crystalline domain. $\varepsilon-\mathrm{Ga}_{2} \mathrm{O}_{3}$ can thus be classified as a pyroelectric material, one more time like III-V nitrides. The symmetry of the $\varepsilon-\mathrm{Ga}_{2} \mathrm{O}_{3}$ structure allows for five distinct piezoelectric coefficients; here we calculate the diagonal coefficient $e_{33}$ as the finite-differences derivative of the polarization with respect to the axial strain $\epsilon_{3}=\left(c-c_{0}\right) / c_{0}$. The result is $e_{33}=0.77 \mathrm{C} / \mathrm{m}^{2}$, which is in line with typical coefficients of strongly polar semiconductors (oxides and nitrides), although over an order of magnitude smaller than those of strong ferroelectrics (see e.g. Ref.13).

These results open up interesting perspectives. The polarization of $\varepsilon-\mathrm{Ga}_{2} \mathrm{O}_{3}$ can be exploited growing the oxide epitaxially on GaN (or, equivalently, growing $\mathrm{GaN}$ on the oxide) to build a high-mobility transistor. The polar axis of $\varepsilon-\mathrm{Ga}_{2} \mathrm{O}_{3}$ is found to be parallel to that of $\mathrm{GaN}$ in growth experiments, ${ }^{77}$ so their polarizations are either parallel or antiparallel. Since the polarization difference is very large in both cases, a correspondingly large polarization charge will appear at the $\mathrm{GaN} / \varepsilon-\mathrm{Ga}_{2} \mathrm{O}_{3}$ inter- face and thus attract free carriers (provided by dopants, e.g.) to form an interface-localized two-dimensional gas at potentially huge concentrations. The polarization difference across the interface, i.e. the polarization charge to be screened by free carriers, and hence the potentiallyreachable local electron-gas concentration, is 0.2 to 0.26 $\mathrm{C} / \mathrm{m}^{2}$, i.e. 1.2 to $1.6 \times 10^{14} \mathrm{~cm}^{-2}$ (for $\mathbf{P}$ vectors in the two materials being parallel or antiparallel, and neglecting possible interface traps, native charges, etc.). In addition, since the gap of $\varepsilon-\mathrm{Ga}_{2} \mathrm{O}_{3}$ is much larger than that of $\mathrm{GaN}$, the interface confinement should be quite efficient. Finally, the sign of the polarization charge and hence of the accumulation layer will depend on the chosen polarity of the substrate. The above scenario is a "writ-large" version of the GaN/AlGaN HEMTs currently in use, whose high-frequency, high-power operation is enabled primarily by the high polarization-induced interface charge. Of course, all else assumed to be equal, $\mathrm{Ga}_{2} \mathrm{O}_{3} / \mathrm{GaN}$ transistors could be much superior to AlGaN/GaN ones, which enjoy a much lower areal density of order $10^{13} \mathrm{~cm}^{-2}$.

\section{SUMMARY}

Using density-functional ab initio theoretical techniques, we have revised the phase diagram of $\left(\mathrm{Ga}_{1-x} \operatorname{In}_{x}\right)_{2} \mathrm{O}_{3}$, showing that the $\beta$ phase is stable (without phase separation into binary components) at low $x$ and exactly at 50-50 concentration; a new hexagonal phase is stable (again without phase separation into binary components) for $x$ from about 0.4 to 0.6 , where it is robustly non-polar; and bixbyite will be favored for $x$ between 0.2 and 0.4 and upward of 0.6 , but should phase-separate into binary components. Around $x \sim 0.5$, the hex, $\beta$ and phase-separated binary bixbyites should be closely competing. Optical signatures are expected at around $4.6 \mathrm{eV}$ at low $x$ ( $\beta$ phase), around $3.5 \mathrm{eV}$ at large $x$ (bixbyite), and at $3.5,4,4.5 \mathrm{eV}$ from the competing phases at $x \sim 0.5$.

We have further studied the $\epsilon$-phase of $\mathrm{Ga}_{2} \mathrm{O}_{3}$, and confirmed it as the second most stable structure beside $\beta-\mathrm{Ga}_{2} \mathrm{O}_{3}$. We find it to have a large spontaneous polarization $\left(0.23 \mathrm{C} / \mathrm{m}^{2}\right)$ and a sizable diagonal piezoelectric coefficient $\left(e_{33}=0.77 \mathrm{C} / \mathrm{m}^{2}\right)$. Symmetry dictates that this phase, once epitaxially stabilized, will not transform back into the ground-state $\beta$, despite having the same volume and a small energy difference; in this sense, the $\varepsilon-\beta$ relation is similar to that between zincblende and wurtzite III-V nitrides.

\section{ACKNOWLEDGMENTS}

Work supported in part by MIUR-PRIN 2010 project Oxide, CAR and PRID of University of Cagliari, Fondazione Banco di Sardegna grants, CINECA computing grants. MBM acknowledges the financial support of her $\mathrm{PhD}$ scholarship by Sardinia Regional Government under 
P.O.R. Sardegna F.S.E. Operational Programme of the Autonomous Region of Sardinia, European Social Fund
2007-2013 - Axis IV Human Resources, Objective 1.3, Line of Activity 1.3.1.
1 M. B. Maccioni, F. Ricci, and V. Fiorentini, Appl. Phys. Express 8, 021102 (2015)

2 M. B. Maccioni, F. Ricci, and V. Fiorentini, J. Phys. Conf. Ser. 566, 012016 (2014).

3 M. B. Maccioni, F. Ricci, and V. Fiorentini, J. Phys.: Condens. Matter 00000000 (2015).

${ }^{4}$ F. Ricci, F. Boschi, A. Baraldi, A. Filippetti, M. Higashiwaki, A. Kuramata, V. Fiorentini, and R. Fornari, J. Phys.: Condens. Matter 00000000 (2015).

${ }^{5}$ H. Peelaers, D. Steiauf, J. B. Varley, A. Janotti, and C. G. Van de Walle, Phys. Rev. B 92, 085206 (2015).

${ }^{6}$ R. Schewski, T. Markurt, T. Schulz, T. Remmele, G. Wagner, M. Baldini, H. von Wenckstern, M. Grundmann, and M. Albrecht, Miscibility and phase separation in the $\left(\mathrm{In}_{x} \mathrm{Ga}_{1-x}\right)_{2} \mathrm{O}_{3}$ system, IWGO-2015 abstract.

7 Y. Oshima, E. G. Villora, Y. Matsushita, S. Yamamoto, and K. Shimamura, J. Appl. Phys. 118, 085301 (2015).
8 S. Yoshioka, H. Hayashi, A. Kuwabara, F. Oba, K. Matsunaga, and I. Tanaka, J. Phys.: Condens. Matter 19 346211 (2007).

${ }^{9}$ Kresse G and Furthmuller J 1996 Phys. Rev. B 5411169.

10 R. D. King-Smith and D. Vanderbilt, Phys. Rev. B 47, 1651 (1993).

11 R. Shannon and C. Prewitt, J. Inorg. Nucl. Chem. 30, 1389 (1968); C. Kranert, J. Lenzner, M. Jenderka, M. Lorenz, H. von Wenckstern, R. Schmidt-Grund, and M. Grundmann, J. Appl. Phys. 116, 013505 (2014); R. Schmidt-Grund, C. Kranert, T. Böntgen, H. von Wenckstern, H. Krauß, and M. Grundmann, J. Appl. Phys. 116, 053510 (2014)

12 D. A. Rusakov, A. A. Belik, S. Kamba, M. Savinov, D. Nuzhnyy, T. Kolodiazhnyi, K. Yamaura, E. TakayamaMuromachi, F. Borodavka, and J. Kroupa, Inorg. Chem. 50, 3559 (2011).

13 W. Jiang, R. Zhang, B. Jiang, and W. Cao, Ultrasonics 41, 55 (2003). 